\title{
Flexibility of nanolayers and stacks: implications in the nanostructuration of clays ${ }^{\dagger}$
}

\begin{abstract}
Tulio Honorio,* Laurent Brochard, Matthieu Vandamme, and Arthur Lebée
The basic structural units of adsorbing microporous materials such as clays and cementitious materials are flexible nanolayers. The flexibility of these layers is reported to play a crucial role in the structuration of these materials, potentially affecting therefore the thermo-mechanical behavior of such materials. Adsorbing fluids are structured in a discrete number of layers within the space between the nanolayers in these materials. This discrete nature of adsorption states may lead to micro-instabilities due to non-convex energy profiles. The transition between adsorption states may involve the bending of layers. Bending contributes to metastability, which is reported to be a potential source of the irreversibilities notably in clay behavior. In this paper, we determine the bending modulus of clays nanolayers by the combination of plate theory with molecular simulations of sodium montmorillonite. The case of clays is illustrative of the behavior of phyllosilicates (i.e. sheet-silicates) which are ubiquitous minerals in Earth's crust. We discuss the conditions in which clay particles, i.e. a stack of nanolayers, can be viewed as thin plates. Estimations of the bending modulus according to the hydration state and dimensions of clay particles are provided. We analyze the implications of the flexibility of the layers in the behavior of a stack of layers as well as in the transitions between adsorption states. The energy barrier associated with bending of clay layers and the characteristic length of bending in such transitions are provided. Our results contribute to a better understanding of the nanostructure of layered adsorbing materials.
\end{abstract}

\section{Introduction}

Nanolayers, i.e. solid layers with thickness on the order of a nanometer and much larger in-plane dimensions, are present in a variety of materials. Several studies have been devoted to understanding or controlling the stability of nanolayers in various applications from acoustic cloaking and energy harvesting to tissue engineering 1 1 6 . Another class of materials that is often also nanostructured in layers are geomaterials such as clays and cement-based materials. The basic structural unit of clay minerals, one of the most abundant material in Earth's crust, are layers with in-plane dimensions of $10 \mathrm{~nm}$ to $20 \mu \mathrm{m}$ and a thickness of circa $1 \mathrm{~nm}^{7}$. Calcium silicate hydrates is the major phase in cement-based materials, which are the most man-made produced material in volume ${ }^{8}$. These silicates are also structured in layers that may present a certain flexibility 9 . Other phyllosilicates (or layered silicates) include micas and serpentines, which are all ubiquitous in nature.

The case of clays can be considered as illustrative of the behav-

Université Paris-Est, Laboratoire Navier (UMR 8205), CNRS, ENPC, IFSTTAR, 6 \& 8 Avenue Blaise Pascal, 77455 Marne-la-Vallée, France.

$\dagger$ Electronic Supplementary Information (ESI) available: Thin and thick plate theory applied to clay layers. See DOI: 10.1039/b000000x/

${ }^{*}$ E-mail: tuliohfaria@gmail.com. Present address: Laboratoire Modélisation et Simulation Multi Echelle, MSME UMR 8208 CNRS, Université Paris-Est, Créteil Cedex 94010, France. ior of phyllosilicates. Locally, in solid clay samples, clay nanolayers are in general structured in ordered (stacked) domains with adsorbed fluid in-between the layers. The stacking process likely stems from the attractive component of the electrostatic interactions modulated by the hydration forces ${ }^{10}$. These locally ordered domains are sometimes designated as crystallites or clays platelets. Experimental results point out a polydisperse distribution of the sizes of clay platelets 11 . At larger scales, a certain degree of disorder often appears, leading to translational and orientational decorrelation ${ }^{12 / 13}$ to the bending of clay layers. The flexibility of clays is deemed to affect the pore sizes in clays 14 , which are in turn associated with key engineering properties such as permeability 15 , diffusivity 1617 , and failure behavior (ductile or brittle) 15 . Furthermore, the possibility of a single clay layer being part of a different particle (i.e. of another domain with different orientational or translational parameters) is reported to be a cause of the structural continuity of larger structural units of clays, such as clay aggregates $\frac{18}{18}$. The effect of the flexibility of the nanolayers constituting these materials on their microstructuration remains unclear.

Indeed, in the literature, both experimental and simulation studies indicate that single clay layers are quite flexible $\frac{19-23}{23}$. Sato et al. ${ }^{19}$, analyzing atomic-force microscopy profiles of thin beidelite layers 24 , observed that a single clay layer deposited over another single or double stacked clay layer bends over a transition length that is less that $50 \mathrm{~nm}$ long. Manevitch et al. ${ }^{21} \mathrm{ob}-$ 
tained, by means of molecular simulations, a bending modulus of $1.25 \times 10^{-17}$ N.m for montmorillonite, with an effective "mechanical" thickness of the clay layer (6.78 A) similar to the distance between the centers of the outermost sheets of atoms of a single layer. Suter et al. $\stackrel{20}{ }$ performed large length scale molecular simulations of clay layers and observed, analyzing the undulatory modes at finite temperature of clay sheets, homogeneous bending in the clay sheet for in-plane lengths above $15 \mathrm{~nm}$. The same authors estimated a flattening transition for an in-plane length above $140 \mathrm{~nm}$. In this case, the resulting bending modulus would be scale-dependent ${ }^{20}$ and the crumpling transition (at longer layers) is also computed. The same authors reported a bending modulus of clay layers of $1.6 \times 10^{-17} \mathrm{~N} . \mathrm{m}$, valid on the range of 15 to $140 \mathrm{~nm}$ of in-plane lengths. Furthermore, the flexibility of nanolayers is directly associated to the change, with respect to the equilibrium state, in the angles between pairs of atomic bonds. The consideration of this kind of three-body interaction is important in the developement of enriched descriptions of material constitutive behavior 2 .

Apart from structural considerations, the flexibility of clay nanolayers might play an important role in the transition occurring between hydration states. Hydration transitions in adsorbing layered materials are a type of "discrete phase transformation" 25 leading to snap-through instabilities. This type of phase transition is at the heart of various phenomena leading to non-linear and inelastic behaviors at the macroscale regarding various materials including alloys, lithium-ion batteries and macromolecular materials (e.g. DNA, spider silk, biopolymers, polysoaps and artificial elastomers) 26|27. Also, swelling clays and cement-based materials are also recognized as materials presenting this kind of snap-through transitions which result in hysteresis at meso- and macro-scales 28 . Given the dimensions of single clay layers, hydration transition presumably involves bending of the layers in such a way that only a limited portion of the layer surmount the energy barrier associated with such transition between two hydration states. Indeed, the flexibility of nanolayers is reported to be a key element in the snap-through behavior of nanolayered materials with non-convex energy, or micro-instabilities 6 . Water (as well as other adsorbing fluids) is structured in a discrete number of layers within the space between clay solid layers. The hydration states are often associated with a specific basal distance $d$. In the case of smectites, dehydrated ( $d=9.7-10.2 \AA)$, mono-hydrated ( $d=11.6-12.9 \AA$ ) , bi-hydrated $(d=14.9-15.7$ $\AA$ ) and tri-hydrated ( $d=18-19 \AA$ ) states as well as a "pore water" state for large pore sizes, can be identified ${ }^{3132}$ (each one of these hydration states is often represented, respectively, by $0 \mathrm{~W}$, $1 \mathrm{~W}, 2 \mathrm{~W}, 3 \mathrm{~W}$ and $\infty \mathrm{W})$. Therefore, the transition between hydration states is associated with volume changes that must be accommodated within the microstructure of swelling clays. The energy barrier associated with transitions involving the whole extension of the clay layer, i.e. not accounting for the flexibility of the layers, have been computed for swelling clays 30 33. 35 .The obtained values well exceed the thermal fluctuations energy, thus swelling clays at this scale are prone to metastability. This metastability is a likely source of irreversibility of the thermo-mechanical behavior of swelling clays. The role of the flexibility of the layers in the metastability of swelling clays is, to the best knowledge of the authors, still to be elucidated. So is the influence of the hydration state on the flexibility of a clay particle.

In this paper, we study the factors affecting the flexibility of clay layers and particles, notably their dimensions and hydration state. The goal is to provide tools adapted to study the effect of the flexibility of phyllosilicates in general from the illustrative case of clays. To do so, we first determine the bending modulus of clays by combining plate theory with molecular simulations for sodium montmorillonite (Na-Mmt) in a multiscale framework. The bending modulus is computed for both in-plane orthogonal directions. We discuss the range of in-plane and out-of-plane dimensions in which clay particles can be viewed as thin plates. Then, we analyze the implications of the found values in the behavior of a single clay layer as well as that of a clay particle. Loads representing stirring, flexure and buckling are examined. Next, we investigate the role of the flexibility of clays in adsorption states transitions. We provide estimations of the energy barriers associated with bending in swelling clays during hydration transitions. The characteristic length in which bending takes place during a transition is also estimated. Finally, we discuss the implications of the layer and particle flexibility on the microtexture of clays. Throughout the article, we provide the scaling laws governing the behavior of clay single layers as well as stacked layers. Pre-

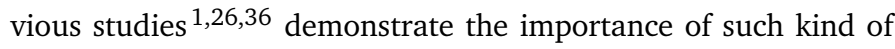
result regarding the behavior of flexible layers or materials presenting non-convex energy landscapes. In these cases, length and time scales associated with phenomena of different physical origins may be superposed in some intervals. Therefore the identification of the associated scaling laws is valuable information in order to understand and control the behaviour of such materials. The results obtained here are easily transferable to the study of other phyllosilicates as we demonstrate in the Section 3.4 .

\section{Models and methods}

\subsection{Flexibility of single clay layers}

Clay layers can be treated as homogeneous sheets bending smoothly provided the length of the layer is much larger than the atomic granularity and much smaller than the persistence length 20 . The atomic granularity corresponds to the typical size of an atom (a few $\AA$ ), while the persistence length is the length for which the sheet crumples under thermal agitation. At scales approaching the atomic granularity, the discrete (atomic) nature of the clay layers must be accounted for. At scales approaching the persistence length, the layers exhibit crumpling transition, i.e., the displacements orthogonal to the basal plane become uncorrelated under the effect of thermal agitation. Crumpling transition occurs when bending energy approaches thermal agitation energy, that is for a length of the order of the ratio between the bending modulus (introduced below) and $k T$. With the estimates of bending moduli provided in this paper, this length widely exceed the maximum length of clay layers. Therefore crumpling transition is not expected for clay layers.

We consider a relaxed clay layer, roughly symmetric with respect to the mid-plane (the asymmetries due to random substi- 


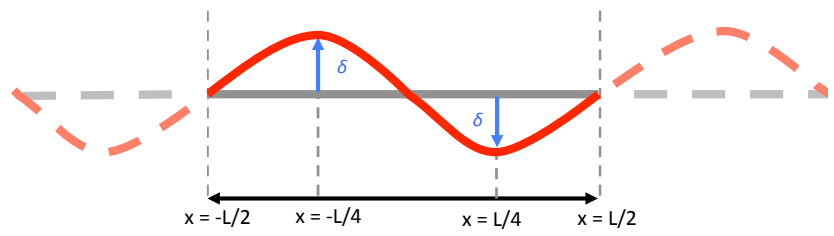

Fig. 1 Periodic plate with period $L$ subjected to punctual forces in a direction $x$ (the forces a constant along $y$ direction)

tutions in octahedral and tetrahedral sheets within the solid layers are neglected), that undergoes no topological changes under moderate loads. At first order, the mechanical behavior is linear elastic and the free energy associated with bending takes the form:

$$
U=\frac{1}{2} \sum_{i, j, k, l \in\{x, y\}} D_{i j k l} \frac{\partial^{2} u_{z}}{\partial i \partial j} \frac{\partial^{2} u_{z}}{\partial k \partial l}
$$

where the $D_{i j k l}$ are the bending moduli; and $u_{z}$ is the displacement orthogonal to the plane of the plate. This expression corresponds to Kirchhoff-Love (or thin-) plate theory which neglects the energy of shear in the $x-z$ and $y-z$ directions. The bending modulus is a key property in the characterization of phyllosilicate nanotexture. In particular, as will be discussed in Section 3.3 the bending modulus is directly related to the propensity of clay to bending in hydration transitions contributing, therefore, to the metastabilities observed in clays. For a thin plate of thickness $h$ made of a homogeneous orthotropic material, the bending moduli of the plate are related to the plane stress stiffness of the material, for instance :

$$
D_{x x x x}=\frac{h^{3}}{12} \frac{E_{x}}{1-v_{x y} v_{y x}}
$$

where $E_{i}$ is the Young's modulus in the $i$ direction and $v_{i j}$ is the Poisson's ratio in the $i$ direction under a load in the $j$ direction. In thin plate theory, the bending moduli $D_{i j k l}$ can be obtained from the variations of energy during a bending test. In this work, to ensure periodic boundary conditions in the molecular simulations, we perform the periodic bending test depicted in Figure 1. For this particular test, the elastic free energy is a quadratic function of the displacement $\delta$ (see Supporting Information):

$$
U=6\left(\frac{4}{L}\right)^{3} D_{x x x x} \delta^{2}
$$

\subsection{Flexibility of a clay particle: a simplified upscaling strat- egy}

The flexibility of a clay particle can be approximated by considering a laminated plate with clay solid layers, with thickness $h_{s}$, intercalated by pore spaces filled by confined electrolyte. Consider a stack of $N$ layers with thickness $h_{\text {tot }}=N\left(h_{s}+h_{w}\right)=N d$, where $h_{w}=d-h_{s}$ is the thickness of the interlayer pore and $d$ is the basal spacing. As detailed in Honorio et al. 30 , in response to a given thermo-mechanical load, clay particles may mix hydration states. This means that, at the scale of a particle an average $\langle d\rangle$, corresponding to a unstable basal distance $d$ at the layer scale, may exist. However, in this paper, we consider that a single hydration state prevails in a stack of layers.

If $h_{\text {tot }} \gg d$, the scale of the clay layer and slit pore can be separated from the scale of the clay particle as sketched in Figure 2 The clay particle can be viewed as made of a homogeneous material. Experimental evidence, according to different techniques (small angle X-ray scattering, X-ray diffraction, transmission electron microscopy and scanning electron microscopy) on a variety of smectites and illites, shows that the average number of solid layers per particle is from few units up to few tens of layers 37.45. In some cases, such as Na-fluorohectorite particles in aqueous solutions, up to 100 solid layers in average can be observed 46 . The distribution of particles' thicknesses can be modeled with a lognormal distribution 12 37/47/48, with the tail of the distribution extending up to a few tens of nanometers 37 . Assuming a few tens of stacked layers, the separability of scales holds and the stack of layers can be viewed as a homogeneous plate at the clay particle scale.

If $L \gg h_{\text {tot }}$, then the assumption of thin plate theory still holds. Regarding in-plane dimensions, clay layers are reported to be equidimensional with sizes ranging from $50 \mathrm{~nm}$ up to $20 \mu \mathrm{m} 7449$. In such large range of particle sizes, the hypothesis of clay particles behaving as thin plates may not be necessarily verified. In the following, we determine the range of sizes for which clay particles can be viewed as thin plates. Indeed, besides particle dimensions, the properties of the confined fluid play a crucial role. Confinement modifies the property of the fluid and, in particular, the confined fluid can support moderate shears and exhibit a non-zero shear modulus. Nonetheless, the elasticity of the confined fluid is orders of magnitudes smaller than that of the solid layers: $\frac{E_{s}}{1-v_{s}^{2}} \gg \frac{E_{w}}{1-v_{w}^{2}}$ and $G_{s}=\frac{E_{s}}{2\left(1+v_{s}\right)} \gg \frac{E_{w}}{2\left(1+v_{w}\right)}=G_{w}$. Although the properties of confined fluids are expected to be anisotropic and heterogeneous because of the layered structure and the distribution of counterions, we assume here an isotropic behavior for simplicity. Indeed any variation of properties at this level is negligible with respect to the high contrast of properties between the fluid and the solid. The same assumption is valid with respect to the heterogeneities (due to isomorphic substitutions) within the solid layer. A stack of clay layers alternates mineral layers and confined water layers. Reasonable estimates of the effective elastic moduli of a stack are obtained by assuming uniform strains in the in-plane directions $x$ and $y$ (Voigt hypothesis) and uniform stress in the orthogonal direction $z$ (Reuss hypothesis). Removing the negligible contributions $\left(E_{w}, G_{w} \ll E_{S}, G_{s}\right)$ one obtains:

$$
\begin{gathered}
E_{x} \approx E_{y} \approx \frac{h_{s}}{h_{s}+h_{w}} E_{s}, v_{x y}=v_{y x}=v_{s} \text { and } G_{x y} \approx \frac{h_{s}}{h_{s}+h_{w}} G_{s} \\
E_{z} \approx \frac{h_{s}+h_{w}}{h_{w}} E_{w} \text { and } G_{x z} \approx G_{y z} \approx \frac{h_{s}+h_{w}}{h_{w}} G_{w}
\end{gathered}
$$

Accordingly, the in-plane behavior is controlled by the solid phase, while the orthogonal behavior is controlled by the confined water. Since the solid and the confined fluid are assumed isotropic, the resulting homogenized medium is trans- 


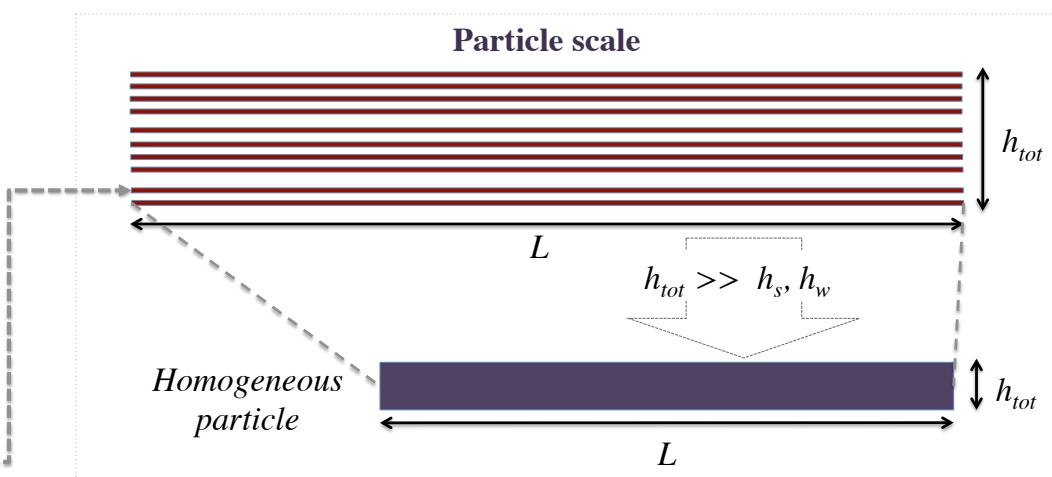

Homogeneous thin layer

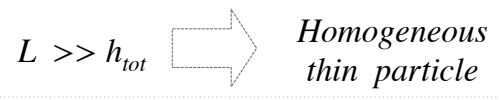

Fig. 2 Separation of scales in clay layer and particle levels: conditions under which the assumptions of homogeneous plate theory still holds.

versely isotropic with a high stiffness contrast: $E_{x}, G_{x y} \gg E_{z}, G_{x z}$. In thin plate theory (Kirchhoff-Love), only the terms associated with strong stiffness $\left(E_{x}, G_{x y}\right)$ are taken into account and contribute to the bending modulus : $D_{x x x x}=D_{y y y y}=\frac{D_{x x y y}}{v_{s}}=\frac{D_{y y x x}}{v_{s}}=$ $\frac{h_{\mathrm{tot}}^{3}}{12} \frac{E_{x}}{1-\left(v_{x y}\right)^{2}}=\frac{h_{\mathrm{tot}}^{3}}{12} \frac{h_{s}}{h_{s}+h_{w}} \frac{E_{s}}{1-v_{s}^{2}}$ and $D_{x y x y}=\frac{h_{\mathrm{tot}}^{3}}{12} G_{x y}=\frac{h_{\mathrm{tot}}^{3}}{12} \frac{h_{s}}{h_{s}+h_{w}} G_{s}$.

Let us consider a plate of length $L$ and bending modulus $D_{x x x x}$ submitted to a longitudinal flexural load $F$ (in out-of-plane $z$-direction) distributed along in-plane $y$-axis. The scaling relation between force and displacement can be deduced by dimensional analysis and takes the general form $\delta \propto \frac{L^{3}}{D_{x x x x}} F$. The multiplicative constant depends on the precise loading conditions. By introducing the expression of $D_{x x x x}$, the scaling laws can be expressed in function of the plate dimensions and mineral elastic modulus only:

$$
\delta_{\text {pure bending }} \propto \frac{L^{3}}{h_{\mathrm{tot}}^{3} E_{S}} F
$$

Kirchhoff-Love's theory neglects the shear orthogonal to the plane $\left(G_{x z}\right.$ and $\left.G_{y z}\right)$. Since the homogeneous medium is very compliant in this direction, it is likely that the plate exhibits shear. Reissner-Mindlin (or thick-plate) theory takes this shear into account in a first-order approximation by assuming constant shear through the thickness of the plate. Two new force resultants appear, namely the shear resultants $\left(Q_{x}\right.$ and $\left.Q_{y}\right)$, conjugated to the angles between the section and the normal to the mid-plane. The elasticity associated with these new resultants are the moduli: $B_{x x}=B_{y y} \propto h_{\mathrm{tot}} G_{x z}=h_{\mathrm{tot}} G_{y z}$. Under a flexural load $F$, by dimensional analysis, the displacement $\delta_{\text {shear }}$ associated with shear only follows the scaling:

$$
\delta_{\text {pure shear }} \propto \frac{L}{B_{x x}} F \propto \frac{L}{h_{\text {tot }} G_{w}} F
$$

Thus, taking into account shear at the first order, the total displacement due to the flexural load takes the form:

$$
\delta \propto \delta_{\text {pure bending }}\left(1+\kappa \frac{E_{s}}{G_{w}}\left(\frac{h_{\mathrm{tot}}}{L}\right)^{2}\right)
$$

where $\delta_{\text {pure bending }}$ is the displacement when shear is neglected
(Eq. 6), and $\kappa$ is a dimensionless constant depending on inital and boundary conditions of the problem. The scaling of Equation 8 shows that shear deformation is significant when the following dimensionless quantity becomes non negligible:

$$
\gamma=\frac{E_{s}}{G_{w}}\left(\frac{h_{\mathrm{tot}}}{L}\right)^{2}
$$

In the limit case of a very thin plate, or of a fairly stiff water layer, $\gamma \ll 1$; so shear can be neglected. In this regime, the behavior of the plate is given by Kirchhoff-Love's theory (Eq. 6). Conversely, in the limit case $\gamma \gg 1$, (i.e. thick plate and very soft fluid layer) the behavior of the plate follows a different scaling given assuming pure shear:

$$
\delta_{\text {pure shear }} \propto \frac{L}{h_{\text {tot }} G_{w}} F
$$

In this case, the behavior of the plate is dictated by the shear modulus of the confined fluid $\left(G_{w}\right)$ and the displacement becomes linear with the aspect ratio $L / h_{\text {tot }}$. As will be discussed in the Results and Discussion section, in the case of clays, both situations are possible.

The profiles of shear stresses $\tau_{x z}$ and $\tau_{y z}$ across the thickness $h$ of a clay particle can be estimated from the static equilibrium of a section. The shear stresses are maximum near the mid-plane and the maximum scales as :

$$
\tau_{x z} \propto \frac{Q_{x}}{h_{\mathrm{tot}}}
$$

where $Q_{x}$ is the shear resultant. For a flexural load $F$ in a 1D problem in $x$ (translational symmetry in $y$ ), the shear resultant scales as $Q_{x} \propto F$ which leads to $Q_{x} \propto \frac{h_{\text {tot }}^{3} E_{s} \delta}{L^{3}}$ in pure bending regime and to $Q_{x} \propto \frac{h_{\text {tot }} G_{w} \delta}{L}$ in pure shear regime.

\subsection{Molecular simulation of clays}

Molecular simulations of clay layers can be used to evaluate the elastic energy $U$ in the bending test of Figure 1 for each in-plane direction of the clay layers. We simulate a layer of sodiumsaturated montmorillonite (Na-Mmt). The atomic structure of montmorillonite is based on the structure of pyrophyllite deter- 

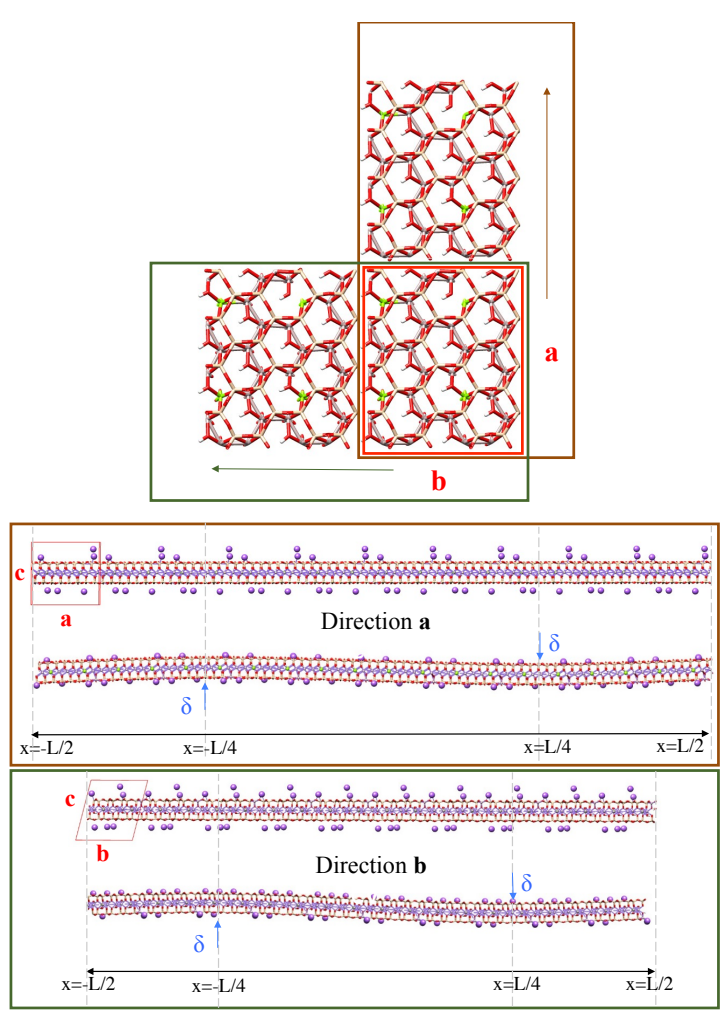

Fig. 3 Snapshot of initial and deformed states (bottom) of clay solid layers under flexion in both directions (top) of the basal plane according to the unit vectors $\mathbf{a}$ and $\mathbf{b}$ of the reference clay supercell. $\mathrm{Na}$ ions are depicted as purple spheres; $\mathrm{O}$ atoms are red, $\mathrm{H}$ white, $\mathrm{Mg}$ green, $\mathrm{Si}$ beige and Al gray.

mined by X-ray diffraction 50 . The reference montmorillonite supercell is obtained by replicating the unit cell of pyrophyllite in each direction of the basal plane. Following Carrier $\frac{51}{1}$, in each layer, one of the 8 octahedral aluminum atoms is substituted by a magnesium atom and one of the 32 tetrahedral silicon atoms is substituted by an aluminum atom. The substitutions follow Loewenstein rule ${ }^{52}$. The total charge density of the layers is $0.124 \mathrm{C} . \mathrm{m}^{2}$, which is compensated by the appropriate number of sodium cations in order to ensure electroneutrality. The resulting structural formula is $\mathrm{Na}_{6}\left[\mathrm{Si}_{62} \mathrm{Al}_{2}\right]\left[\mathrm{Mg}_{4} \mathrm{Al}_{28}\right] \mathrm{O}_{160}(\mathrm{OH})_{32} \cdot n \mathrm{H}_{2} \mathrm{O}$. This reference supercell has in-plane dimensions $L_{x}=21.18 \AA$ and $L_{y}=18.28 \AA$ and a thickness $L_{z}=6.56 \AA$.

Following the results of Ngouana W. and Kalinichev [53, we assume that the specific locations of isomorphic substitutions has little effects on the thermodynamic and structural properties of the system (especially in the case of the studied Na-Mmt with moderate structural charge and a monovalent counterion).

To apply plate theory, one has to consider a thin layer. We replicate the reference supercell of Na-Mmt 10 times in the direction of unit cell vector $\mathbf{a}$ and in the direction orthogonal to a (direction b). Figure 3 (top) displays both directions. Note that the in-plane tilt, that is the tilt with respect to unit cell vectors $\mathbf{a}$ and $\mathbf{b}$ for the reference cell is small $(0.018 \AA)$ and therefore $\alpha \approx 90^{\circ}$. Thus, the direction orthogonal to $\mathbf{a}$ is approximately parallel to the unit cell vector $\mathbf{b}$. The aspect ratio of the simulated layers for each di- rection are, respectively, $L_{z} / L_{x}=0.039$ (dir. a) and $L_{z} / L_{y}=0.036$ (dir. b), which justifies the application of the thin plate theory.

The simulations are performed with LAMMPS software ${ }^{54}$ in the canonical ensemble (NVT) at $0.1 \mathrm{~K}$ with a Nosé-Hoover thermostat. The interactions between atoms are described by LennardJones and Coulombic interactions. The Lennard-Jones parameters and the partial charges are obtained from the ClayFF force field ${ }^{55}$. Lennard-Jones parameters for cross interactions are derived following Lorentz-Berthelot mixing rules. A cutoff distance of $8.9 \AA$ is adopted for the short range dispersive interactions. We employ the Ewald sum method for long range electrostatic interactions with a relative precision of $10^{-5}$ in forces. Simulations are run with periodic boundary conditions, required by the Ewald sum implementation. To reduce the influence of periodic images on the behavior in the direction orthogonal to the plane of the layer, $4 \mathrm{~nm}$ are added to the length of the simulation box in that direction. The ratio of the total extended volume compared to the volume of a simulation box (corresponding to a basal space of $12 \AA(1 W)$ ) is 3.3, thus limiting the electrostatic interactions between parallel periodic replicas to negligible values.

For each simulation point (in both in-plane directions), a displacement $\delta_{i}=\delta_{i-1}+\Delta \delta_{i}$, with $\Delta \delta_{i}=0.02 \AA$, is imposed at a distance of one-quarter box from each edge of the simulation box. For each increment $\Delta \delta_{i}$, a 5 ps-simulation is run and the potential energy $U_{i}$ is sampled during the last $1 \mathrm{ps}$. The final configuration of the previous simulation $\left(\delta_{i-1}\right)$ is used as the initial configuration. The elastic bending energy $U_{i}$ is determined by the difference between the potential energy $W_{0}$ of the relaxed state and the potential energy $W_{i}$ associated with the displacement $\delta_{i}$, i.e. $U_{i}=W_{0}-W_{i}$. Figure 3 (bottom) shows relaxed and deformed configurations for both $\mathbf{a}$ and $\mathbf{b}$ directions.

In order to account for the influence of the position of counterions (here, forming inner-sphere surface complexes), ten different configurations are tested, each one with $\mathrm{Na}$ ions being randomly located in the free space of the simulation box. The standard deviation in the elastic energy is provided with respect to these 10 simulations. We do not account for the interlayer water and its likely screening effect. Manevitch and Rutledge 21 also performed molecular simulations of clay in vacuum. The values of bending modulus and in-plane Young modulus obtained by these authors are in fair agreement with the simulations explicitly accounting for water and with larger clay layers 20 . However, it is argued in the literature $\underline{20}$ that the presence of interlayer water molecules and counterions may lead to stiffer clay layers.

\section{Results}

First, we present the estimations of the bending modulus of a single mineral layer of clay. With these estimations, some scenarios can be studied in order to get insights into the behavior of clay particles. Here, we consider two cases: (1) the flexibility of a single clay layer and of a clay particle (i.e. a stack of layers) and (2) the characteristic length in which a transition of hydration state occursin swelling clays. 


\subsection{Bending modulus of a single clay layer}

Following Eq. 3, the bending moduli in the in-plane directions are estimated from the elastic energies $U_{\mathbf{a}}$ and $U_{\mathbf{b}}$ (Figure 4left) obtained by molecular simulations. The results are shown in Table 1. The values are in agreement with the bending moduli of clays estimated by other authors: $1.6 \times 10^{-17} \mathrm{~N} . \mathrm{m}$ by Suter et al. ${ }^{20}$ and $1.25 \times 10^{-17}$ N.m by Manevitch and Rutledge ${ }^{21}$. As also observed by Sato et al. 19, the clay layer is found to be more flexible in the $\mathbf{a}$ direction than in the $\mathbf{b}$ direction. Both bending moduli have the same order of magnitude, which is consistent with the observation that the flexibility of a clay layer, in both in-plane directions, is related to the broadening of the distribution of Si-O-Si angles within tetrahedral sheets $\frac{19}{19}$. In our simulations, the positions of $\mathrm{Na}$ counterions are responsible for a standard deviation of less that $10 \%$ of the bending modulus.

The Young modulus of a single layer, in each in-plane direction, can be obtained by adopting an effective thickness of the clay layer $h_{s}$ in Equation 2. The in-plane Poisson ratios of clay layers are reported to be approximately $v_{\mathbf{a b}} \approx v_{\mathbf{b a}} \approx 0.36^{20}$. The effective thickness can be chosen according to two effective 'mechanical' thicknesses of the solid layers:

- a) $h_{s}=6.42 \AA$, which corresponds, in this study, to the distance between the center of the out-most $\mathrm{O}$ atoms in each surface of the solid layer.

- b) $h_{s}=9.10 \AA$, which corresponds to $6.42 \AA$ plus the twice the van der Waals radius of O atoms $\left(r_{O}^{V D W}=1.34 \AA\right)^{20}$.

Similar reasoning was used by Suter et al. $\underline{20}$. The Young modulus found here for $h_{s}=9.10 \AA$ is closer to the values found by these authors considering the van der Waals radius of $\mathrm{O}$ atoms in the effective thickness of the solid layers. With $h_{s}=6.42 \AA$, the bending modulus of the clay particles are roughly 3 times that of the case with effective thickness $h_{s}=9.10 \AA$. For the Young moduli of Table 1 the positions of the Na counterions are responsible for a standard deviation of less that $10 \%$.

Figure 4 (right) displays the components $C_{i i i i}$ (with $\mathrm{i}=\mathbf{a}$,b) of the stiffness tensor of a clay particle computed with both values of $h_{s}$ and compared to values reported in the literature5657. In our estimations, we employed the range of basal spacings, mentioned in the introduction of this paper, corresponding to the various hydration states as reported by Ferrage et al. 31 . The in-plane elastic properties of clay particles computed considering $h_{s}=9.10 \AA$ are closer to the values reported in the literature. This effective "mechanical" thickness is retained in the following in the computation of the bending moduli of clay particles.

With the estimates of bending moduli, we can discuss the flexibility of a single clay layer. Apart from the crumpling transition that we already discarded, a plate can exhibit two main types of out-of-plane deformations, namely bending and buckling instability. Which of the two mechanisms prevails depends on the length of the plate and on the magnitude of loading. In thick plate theory, the critical axial force triggering buckling takes the form $F_{c}=\left(\frac{L^{2}}{\pi^{2} D}+\frac{1}{B}\right)^{-1}[58$, where the first term corresponds to the pure bending regime and the second term to the pure shear regime. Therefore, in pure bending regime, which is the case of a single clay layer, there exists a critical length of the plate below which bending always prevails over buckling: $L_{c}=\pi \sqrt{\frac{D}{F}}$. Considering force magnitudes corresponding to stirring in solution $\left(F \sim 10 \mathrm{kPa} \times h_{s}\right)$, the critical length is about $5 \mu \mathrm{m}$ and buckling is not expected even for the longest layers. Considering force magnitudes corresponding to geomechanical conditions, $\left(F \sim 1 \mathrm{MPa} \times h_{s}\right)$, the critical length is about $500 \mathrm{~nm}$. Buckling is theoretically possible for a layer free of constraints over this length ${ }^{22}$, which is unlikely since clays are usually densely packed in geomechanical conditions. Therefore bending is expected to be the major type of flexural deformation of clay layers. This conclusion remains true for stacks of layers with higher bending moduli.

Let us consider the bending deformation of a clay layer submitted to a distributed out-of-plane loading $f$. The mechanical behavior of a single clay layer is well described by the pure bending regime and the out of plane displacement scales as follows:

$$
\delta \propto \frac{f \cdot L^{4}}{D}
$$

Molecular simulations of clay layers with reactive force fields ${ }^{19}$ show that the solid layers can support curvatures with radius of bending as low as $1 \mathrm{~nm}$ and stresses up to $800 \mathrm{MPa}$ before fracture. In the case of a single clay layer, for a range of pressures corresponding to vigorous stirring $\left(\approx 10^{3}\right.$ to $\left.10^{5} \mathrm{~Pa}{ }^{59}\right)$, the scaling law (12) is shown in Figure 5 The displacement $\delta$ is of the order of $L$ for clay layers with length $L$ exceeding $100 \mathrm{~nm}$. Therefore, a single (exfoliated) clay layer can exhibit a highly bent form even due to stirring in solution.. Experimental observations suggest that suspesions of $\mathrm{Li}^{+}$and $\mathrm{Na}^{+}$exchanged smectites present exfoliated layers $10 / 43$, and this occurs even if the multiple hydrodynamical interactions tend to put particles parallel to each other.

\subsection{Bending of a stack of layers}

As discussed in Section 2.2, out-of-plane shear may or may not be negligible for the bending of clay particles depending on the interplay between the aspect ratio of the stack $h_{\text {tot }} / L$ and the contrast between the elasticity of the solid layer and that of the fluid $E_{s} / G_{w}$. For the $E_{s} / G_{w}$ ratio, it is necessary to well define the mechanical properties of the confined fluid phase, notably with respect to its shear bearing capacity. The behavior of confined fluids often differs from the behavior of bulk fluids. Confined water is reported to present solid-like and glassy behaviors in pores of circa $1 \mathrm{~nm}$ 60. Due to the structuration of water in clay interlayer pores, a certain resistance to shear can emerge. Indeed, the elastic tensor computed at the layer scale with molecular simulations ${ }^{56}$ identified a non-zero shear stiffness in out-plane directions, which can be used to estimate the shear moduli of the confined electrolyte (see Tab. 2). Considering these values, the ratio $E_{s} / G_{w}$ varies from roughly 200 to 3000 .

The dimensionless number $\gamma$ (Eq. 9) determines whether the shear of interlayer water dictates the behavior $(\gamma \gg 1$ - pure shear regime) or whether shear can be neglected ( $\gamma \ll 1$ - pure bending regime). Figure 6 (left) displays a chart of in-plane length $L$ and number of stacks $N$ of a clay particle for which $\gamma=1$, i.e. $h_{\text {tot }} / L=$ $\sqrt{G_{w} / E_{s}}$ for the various hydration states $0 \mathrm{~W}, 1 \mathrm{~W}, 2 \mathrm{~W}$ and $3 \mathrm{~W}$. 

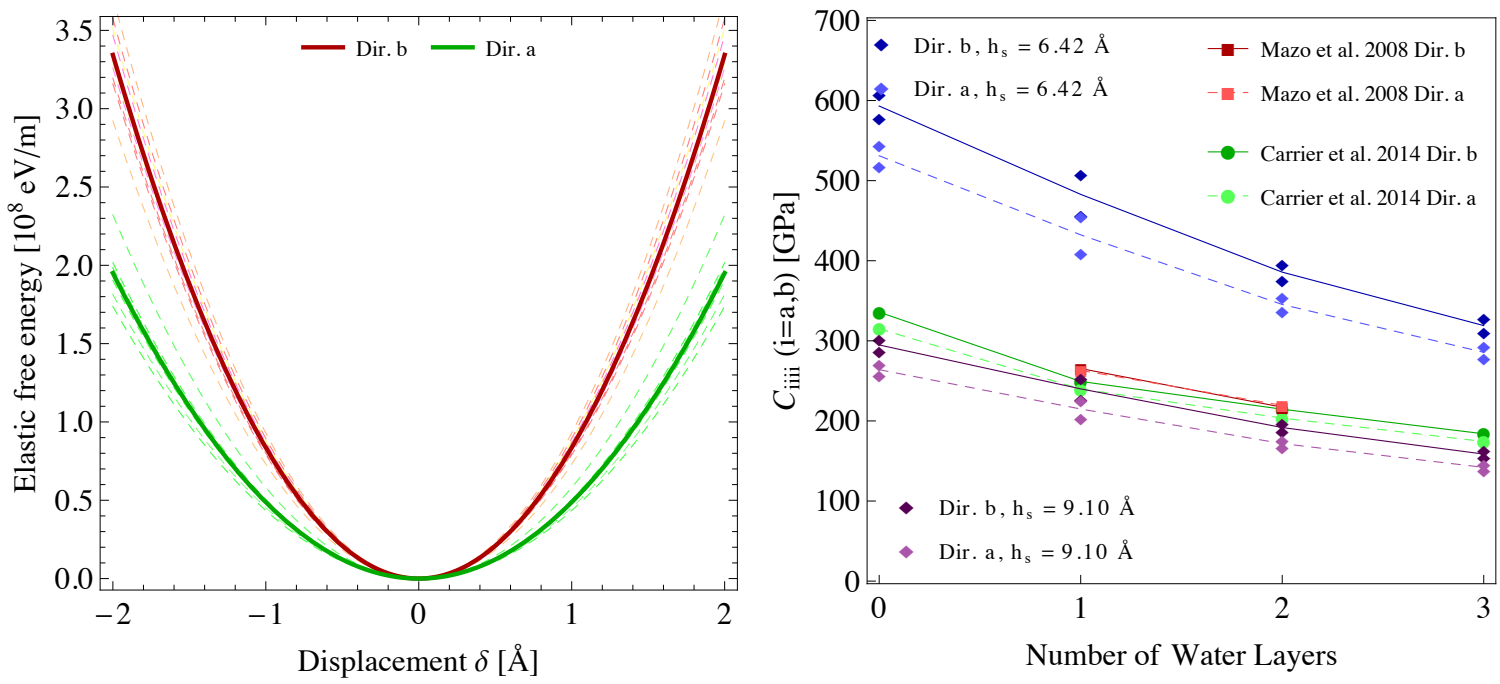

Fig. 4 At left, elastic energy $U_{\mathbf{a}}$ and $U_{\mathbf{b}}$ obtained from molecular simulations. The dashed curves are the results of each one of the 10 simulations with different initial positions of $\mathrm{Na}$ counterions; the solid curves are the average for each direction. At right, components $C_{i i i i}$ (with $\mathrm{i}=\mathbf{a}, \mathbf{b}$ ) of stiffness tensor of clay layers computed by molecular simulations. The curves from this work (purple lines for $h_{s}=9.10 \AA$ and blue lines for $h_{s}=6.42 \AA$ ) are obtained by averaging the estimates corresponding to the lower and upper values of basal spacing associated with the hydration states of smectites: OW $(d=9.7-10.2 \AA), 1 \mathrm{~W}(d=11.6-12.9 \AA), 2 \mathrm{~W}(d=14.9-15.7 \AA)$ and $3 \mathrm{~W}(d=18-19 \AA) 31$. Dashed lines correspond to direction a and full lines, to direction $\mathbf{b}$.

Table 1 Bending and Young moduli in the $\mathbf{a}$ and $\mathbf{b}$ directions for a single Na-Mmt layer. The Young moduli are obtained from Eq. 2 Two effective thicknesses $h_{s}$ are considered: a) the distance between the centers of the outermost oxygen atoms of both surface of a single layer after relaxation ( $h_{s}$ $=6.42 \AA)$ and $\mathrm{b}$ ) this same distance plus twice the van der Waals radius ${ }^{20}$ of oxygen atoms $\left(h_{s}=9.10 \AA\right)$

\begin{tabular}{cccc}
\hline Direction & $D_{\text {iiii }}\left(\times 10^{-17} \mathrm{~N} . \mathrm{m}\right)$ & $E_{S}\left(h_{s}=6.42 \AA\right) \mathrm{GPa}$ & $E_{S}\left(h_{s}=9.10 \AA\right) \mathrm{GPa}$ \\
\hline $\mathbf{a}$ & $1.811 \pm 0.147$ & $714.8 \pm 58.0$ & $251.0 \pm 20.4$ \\
\hline $\mathbf{b}$ & $2.023 \pm 0.140$ & $798.5 \pm 55.2$ & $280.4 \pm 19.4$ \\
\hline
\end{tabular}

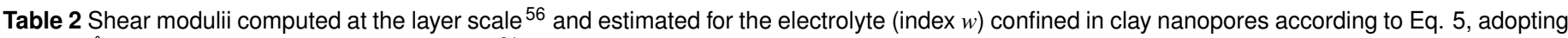
$h_{s}=9.10 \AA$ and the experimental basal spacings 31 .

\begin{tabular}{cccccc}
\hline Direction & & $0 \mathrm{~W}$ & $1 \mathrm{~W}$ & $2 \mathrm{~W}$ & $3 \mathrm{~W}$ \\
& & $d=9.7 \AA$ & $d=11.6 \AA$ & $d=14.9 \AA$ & $d=18.0 \AA$ \\
\hline \multirow{2}{*}{$\mathbf{a}$} & $G_{\mathbf{a} z}(\mathrm{GPa})$ & 10.6 & 1.7 & 1.6 & 0.2 \\
\cline { 2 - 6 } & $G_{\mathbf{a} z, w}(\mathrm{GPa})$ & 0.65 & 0.36 & 0.62 & 0.09 \\
\hline \multirow{2}{*}{$\mathbf{b}$} & $G_{\mathbf{b} z}(\mathrm{GPa})$ & 18.5 & 1.9 & 0.3 & 0.9 \\
\cline { 2 - 6 } & $G_{\mathbf{b} z, w}(\mathrm{GPa})$ & 1.14 & 0.40 & 0.11 & 0.44 \\
\hline
\end{tabular}




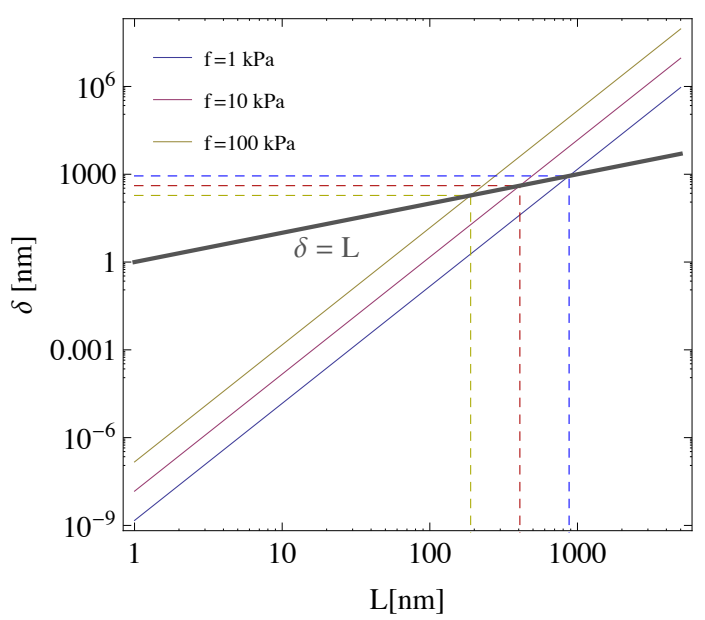

Fig. 5 Scaling of the out-of-plane displacement for a single layer submitted to distributed forces of amplitude $f$ (Eq. 12). The line $\delta=L$ displays the limit of highly deformed configuration, for which the theory assuming small deformation is no more valid. We consider the bending modulus in direction $\mathbf{b}$ (Tab. 1 .

The range of values considered in this chart is representative of the observed length and number of clay layers. According to this chart, clay particles can present either deformation regimes: pure bending above the line, and pure shear below.

Figure 6 (center and right) shows the scalings of out-of-plane displacement $\delta$ (Eqs. 6land 10). Figure 6 (center) shows the scaling in function of the length $L$ for two number of stacks $N$, and Figure 6 (right) shows the scaling in function of the number of stacks $N$ for two in-plane lengths $L$. Both regimes, related to pure bending and pure shear approximations, are relevant for the dimensions of clay particles considered. Particles with few long layers behave in pure bending regime (scaling in $L^{3}$ and $N^{-3}$ ), and particles with many shorter layers behave according to pure shear regime (scaling in $L$ and $N^{-1}$ ). The transitions between these two regimes depend on the dimensions of the clay particles and on the hydration state. To get the measure of the quantity $\delta \times\left(E_{S} / F\right)$ displayed in Figure 6, one can consider the case of geomechanical applications for which $F / L$ is of the order of $1 \mathrm{MPa}$ : in that case, $E_{S} / F \sim 10^{11} \mathrm{~m}^{-1}$ for $L \sim 1 \mu \mathrm{m}$ and $\delta \times\left(E_{S} / F\right) \sim 10^{11} \delta$ in units of $\mathrm{m}$. Therefore, values of $\delta \times\left(E_{S} / F\right)$ exceeding $10^{5}$ correspond to very significant deformations $(\delta \sim L)$ under geomechanical loading. According to Figure 6, such a level of deformation is possible only in pure bending regime for stacks less than 20 layers thick and more than $0.5 \mu \mathrm{m}$ long.

Let us define an effective bending stiffness of a clay particle as the ratio between the loading force and the associated displacement : $F / \delta$. In pure bending regime, the effective stiffness is $F / \delta \propto D / L^{3}$, while in the pure shear regime it takes the form $F / \delta \propto B / L$. Since the moduli $D$ and $B$ strongly depends on the total thickness $h_{\text {tot }}=N d$, one expects a dramatic increase of the effective stiffness with $N$. The effect of the basal spacing $d$ is more complex to anticipate since the material elasticity depends on the hydration state. The scaling of the effective stiffness in the two regimes can be rewritten :

$$
\begin{gathered}
\text { pure bending regime }: \frac{F L^{3}}{\delta D_{0}} \propto \frac{N^{3} d^{2}}{h_{s}^{2}} \\
\text { pure shear regime }: \frac{F L^{3}}{\delta D_{0}} \propto \frac{1}{\gamma^{\prime}} \frac{N d}{h_{s}}
\end{gathered}
$$

where $D_{0}=\frac{h_{s}^{3}}{12} E_{S}$ is the bending moduli of a single solid layer and $\gamma^{\prime}=\frac{1}{12}\left(\frac{h_{s}}{L}\right)^{2} \frac{E_{s}}{G_{w}}$. We display the scaling of the effective bending stiffness in Figure 7 for $L=100$ and $500 \mathrm{~nm}$ and $N=10$ and 50. Only the basal spacings corresponding to stable hydration states, mentioned in the introduction of this paper as reported by Ferrage et al. 31 , are considered. In all cases, including both pure bending and pure shear regimes, a stack of layers is less flexible than a single clay layer $\left(\left(F L^{3}\right) /\left(\delta D_{0}\right)=1\right)$. The particle with $N=10$ and $L=500 \mathrm{~nm}$ is in pure bending regime for $0 \mathrm{~W}$ to $2 \mathrm{~W}$ hydration states. In pure bending regime, the effective bending stiffness increases with the basal spacing or number of water layers in a pore. Increasing the number of solid layers leads to a pure shear response associated with hydration states with higher water content. In pure shear regime, the scaling of effective bending with the basal spacing is complex since the hydration state affects the magnitude of the shear modulus $G_{w}$. Since the shear modulus $G_{w}$ of confined water reported in 51 does not evolve monotonously with the hydration state (Tab. 2), in pure shear regime the resulting effective bending stiffness is not monotonous with respect to the hydration state. Note however that this observation is questionable since the estimation of $G_{w}$ is not accurate, and more precise estimates could well decrease monotonously with the hydration state. According to Figure 7 the effective bending stiffness of a clay particle appears very sensitive to the size L and number of layers $N$ but less sensitive to basal spacing $d$ and changes in hydration state.

In the case of a stack of layers, the energy of bending and displacement scales with $N^{3}$ (pure bending) or $N$ (pure shear) depending on the particle dimensions and hydration state. We can estimate that even for large clay particles (e.g. $L=1000 \mathrm{~nm}$ ) under vigorous stirring, for a stack of few tens of clay solid layers, the maximum $\delta$ is in the order of a nm or less. So, clay particles exhibit very little bending in disperse solutions, in contrast with the case of single exfoliated layers.

Shear stresses induced by bending within clay layers and particles can be estimated using Eq. 11. These shear stresses may induce losses in translational correlation (i.e. the solid layers keep the same orientation but move in an in-plane direction) between the layers or delamination of clay particles according to their in-plane dimensions, number of stacks and hydration state. Carrier ${ }^{51}$ simulated shear of clay layers and observed that the viscous behavior of a stack of layers corresponds to a yield stress fluid: for stresses below a yield stress $\tau_{c}$, no irreversible deformation is observed; whilst, for stresses above $\tau_{c}$, the shear rate is proportional to the difference between the shear stress and $\tau_{c}$. The values of $\tau_{c}$ estimated by Carrier 51 for Na-Mmt are given in Table 3. These values indicate the maximum shear stresses that can be supported by the interlayer water in a clay particle before irreversible deformations of the stacks take place. 

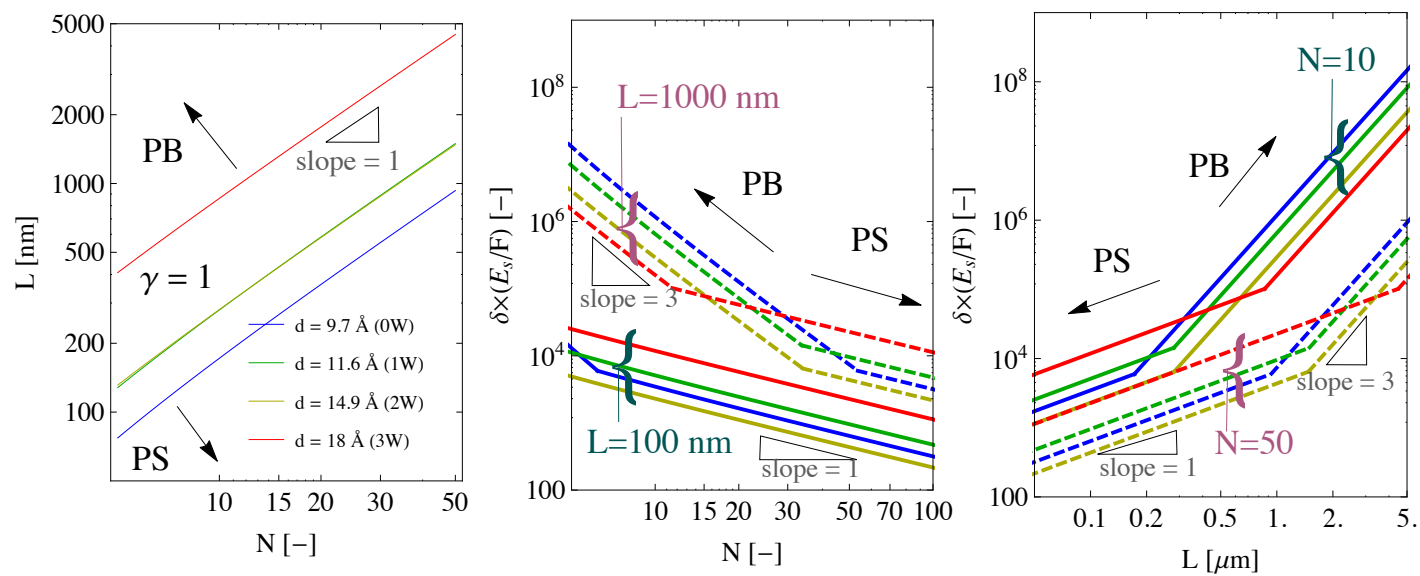

Fig. 6 At left, in-plane length $L$ and number of stacks $N$ of a clay particle for which the dimensionless quantity $\gamma=1$ according to the hydration state. Scaling of the out-of-plane displacement $\delta$ with the number of stacks $N$ (at center) and with the in-plane length $L$ (at right). PB denotes pure bending regime (Eq. 6); and PS, pure shear regime (Eq. 10. We consider the values of $G_{w}$ and $E_{s}$ provided in Tables 2 and 1 respectively (direction a with $\left.h_{s}=9.10 \AA\right)$. Note that PB and PS regimes are asymptotic regimes, and the actual transition between these regimes is smooth.

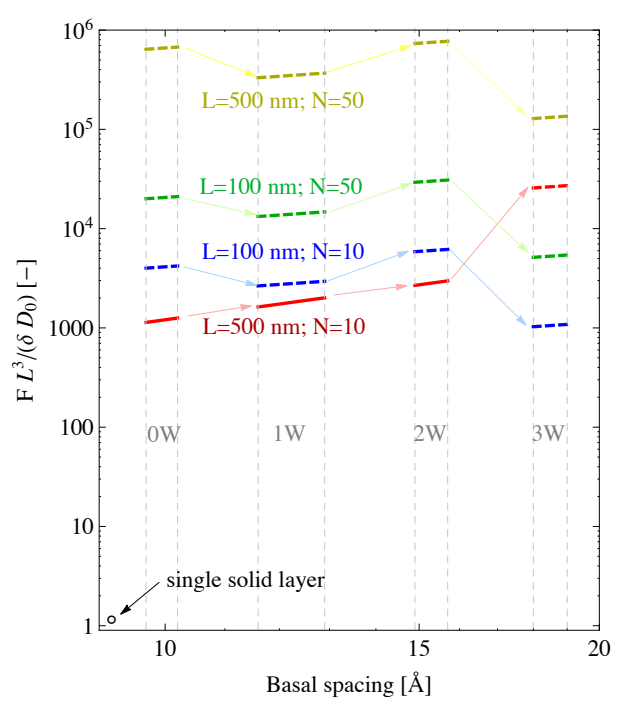

Fig. 7 Dimensionless effective bending stiffness as a function of the basal spacing $d$ for $L=100$ and $500 \mathrm{~nm}$ and $N=10$ and 50 solid layers. The range of basal spacings associated with $0 \mathrm{~W}, 1 \mathrm{~W}, 2 \mathrm{~W}$ and $3 \mathrm{~W}$ hydration states are shown 31 . Pure bending regime is identified by the full lines, and pure shear regime by the dashed lines. We consider the values of $G_{w}$ and $E_{s}$ provided in Table 2 and 1 (direction a with $h_{s}=9.10 \AA$ ).
Table 3 Yield stress of the confined fluid under shear in Na-Mmt

\begin{tabular}{c|ccc} 
Hydration state & $1 \mathrm{~W}$ & $2 \mathrm{~W}$ & $3 \mathrm{~W}$ \\
\hline$\tau_{c}(\mathrm{MPa})$ & $18.5 \pm 1.2$ & $1.0 \pm 1.1$ & $0.5 \pm 0.3$ \\
\hline
\end{tabular}

When a clay particle is submitted to distributed forces of magnitude $f$, the shear resultant is of the order of $Q \propto f L$ and does not depend on the regime of deformation (pure bending or pure shear). Therefore the shear stress within the clay particle (Eq. 11) scales as :

$$
\tau \propto \frac{f L}{N d}
$$

Note that, under displacement control, the scaling differs between pure bending and pure shear regimes. However, displacement control is irrelevant when comparing particles of different dimensions because of the huge difference in bending stiffness. Accordingly, we limit ourselves to loading in force. We display the scaling of Equation 15 in Figure 8 for moderate distributed forces ( $f=10 \mathrm{kPa}$ ) corresponding to stirring of a suspension and for strong distributed forces ( $f=1 \mathrm{MPa}$ ) corresponding to geomechanical conditions. We considered two particle lengths $L=100$ $\mathrm{nm}$ and $L=1000 \mathrm{~nm}$ and all the hydration states (0W, 1W, 2W and $3 \mathrm{~W}$ ). We also display in Figure 8 the yield stresses of the confined fluid (Tab. 3). The shear stress decreases significantly with the hydration state and with the number of layers. The shear resulting from stirring $(f=10 \mathrm{kPa})$ may exceed the yield stresses of 


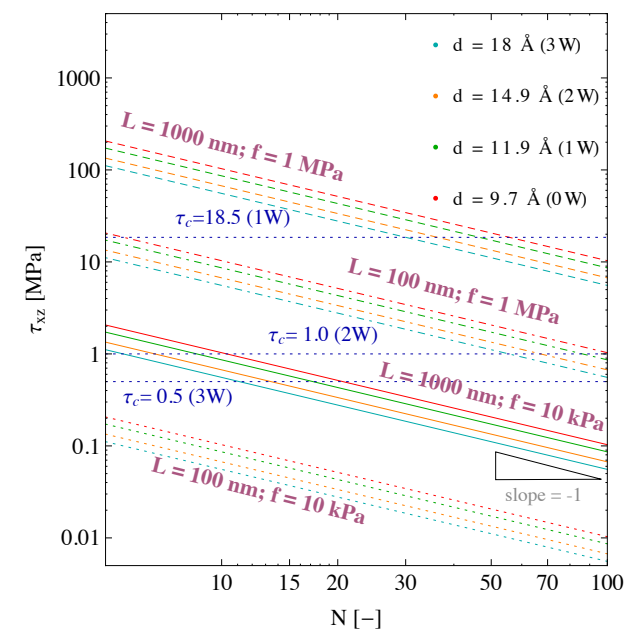

Fig. 8 Scaling of the shear stresses $\tau$ (Eq. 15 as a function of the number of solid layers $N$, for two lengths $(L=100 \mathrm{~nm}$ and $L=1000 \mathrm{~nm})$ and two loadings ( $f=10 \mathrm{kPa} \mathrm{nm}$ and $f=1 \mathrm{MPa})$. The yield stresses (Tab. 3 of the different hydration states are displayed.

2W and 3W clay particles with $L=1000 \mathrm{~nm}$ and $N<20$ solid layers; in this case, stirring seems to be enough to induce irreversible deformations in particles. The shear resulting from a geomachanical load stirring ( $f=1 \mathrm{MPa}$ ) may exceed the yield stresses of $2 \mathrm{~W}$ and 3W for particles with clay particles with $N<70$ solid layers $L>100 \mathrm{~nm}$. In the specific case of long layers $(L=1000 \mathrm{~nm})$ and strong distributed forces, irreverssible deformations inducing translational decorrelation of clay particles also occur for $1 \mathrm{~W}$ state and $N<50$ solid layers.

\subsection{Bending during transitions between hydration states}

An interesting question is the role of clay flexibility in the transitions between hydration states in swelling clays. An energy barrier of few $k T$ per $\mathrm{nm}^{2}$ separates stable hydration states in swelling clays $30 \mid 33$. Since the size of clay layers ranges from tens of nanometers up to tens of micrometers, transition presumably involves bending of the layers in such a way that only small portions of the layers overcome that energy barrier. To investigate this question, one has to estimate how the bending energy compares to the energy barrier. Metastability is claimed to play an important role in the irreversibilities observed in swelling clays' thermo-mechanical behavior 3361,64 . Since bending controls the overall energy barrier between hydration states, it may play a major role in the occurrence of metastability during the hydration transitions.

Let us consider the hydration transition from $(n+1) W$ to $n W$ state sketched in Figure 9 (a). We assume symmetry with respect to the dashed horizontal and vertical lines so that we can limit our analysis to the study of a single half-layer. The upper surface of the layer is subjected to an uniformly distributed imposed pressure $P_{\text {control }}$, while the lower surface is subjected to a non-uniformly distributed pressure $P(\delta)$ that depends on the half basal spacing $\delta=d / 2$. Static equilibrium requires:
$P_{\text {control }}=\frac{1}{L} \int_{0}^{L} P(\delta(x)) d x$. To estimate the minimal energy barrier needed to accomplish the transition $(n+1) W$ to $n W$, we make the half basal spacing $\delta(0)$ evolves from the value corresponding to hydration state $(n+1) W$ to the value corresponding to $n W$. The system adopts the configuration minimizing the thermodynamic potential: $\Lambda=\Phi-\mu_{w} N_{w}+\int_{0}^{L} P_{\text {control }} \delta(x) d x$, where $\Phi$ is the Helmholtz free energy and $\mu_{w}$ is the chemical potential of water. At fixed temperature and water chemical potential, there are two contributions to this energy: that of the hydration and that of the bending of the clay layer. These two contributions are:

$$
\Lambda=\Lambda_{\text {hyd }}+\Lambda_{\text {bend }}=\int_{0}^{L} \lambda_{P_{\text {control }}}(\delta(x)) d x+\frac{D}{2} \int_{0}^{L}\left(\frac{d^{2} \delta}{d x^{2}}\right)^{2} d x
$$

where $\lambda_{P_{\text {control }}}(\delta)$ is referred to as the swelling free energy 30 [33/35 and can be obtained by integration of the confining pressure isotherms over the basal spacing. Molecular simulation can be used to compute these pressure isotherms. A transition of hydration state involves an energy barrier $\Delta \lambda_{P_{\text {control }}}$ of a few $k T$ per $\mathrm{nm}^{2}$ when the control pressure is the pressure of stable transition $\left(\lambda_{P_{\text {stable }}}\left(\delta_{(n+1) W}\right)=\lambda_{P_{\text {stable }}}\left(\delta_{n W}\right)\right)$. As $P_{\text {control }}$ increases, the energy barrier decreases (see ${ }^{30}$ ).

Without going as far as solving this mechanical problem in detail, one can already provide some estimations by comparing the magnitudes of the two contributions (namely, hydration transition and bending). Let us assume that the hydration transition occurs over a length $l_{\text {trans }}$ (depicted in Figure 9(b)). The hydration energy barrier is of the order $\Delta \lambda_{P_{\text {control }}} \cdot l_{\text {trans }}$, whereas the bending energy is of the order $\frac{D}{2} \cdot\left(\frac{\Delta \delta}{l_{\text {trans }}^{2}}\right)^{2} \cdot l_{\text {trans }}$, where $\Delta \delta$ is the difference of basal spacing between the two hydration states. Minimization of the total energy $\Lambda$ requires $\partial \Lambda / \partial l_{\text {trans }}=0$, which leads to the following estimates of the length of hydration transition :

$$
l_{\text {trans }} \sim\left(\frac{(\Delta \delta)^{2} D}{\Delta \lambda_{P_{\text {control }}}}\right)^{1 / 4}
$$

In the limit $l_{\text {trans }}>L$, the bending energy dominates the mechanical behavior. The layer is very rigid and very little bending is observed. Therefore, the energy barrier per unit length in the orthogonal $y$ in-plane direction, associated with the hydration transition has the order of magnitude of $L \Delta \lambda_{P_{\text {control }}}$. In contrast, in the limit $l_{\text {trans }} \ll L$, the layer is very flexible and deforms within a short distance compared to the length of the clay layer. The resulting energy barrier has, then, the order of magnitude of $l_{\text {trans }} \Delta \lambda_{P_{\text {control }}}$. Accordingly, one can determine which of the two regimes applies by estimating the dimensionless quantity:

$$
\frac{l_{\text {trans }}}{L}=\left(\frac{(\Delta \delta)^{2} D}{L^{4} \Delta \lambda_{P_{\text {control }}}}\right)^{1 / 4}
$$

The energy barriers $\Delta \lambda_{P_{\text {control }}}$ for $0 \mathrm{~W} / 1 \mathrm{~W}$ and $1 \mathrm{~W} / 2 \mathrm{~W}$ transitions are of the order of a few $\mathrm{kT} / \mathrm{nm}^{2}$. The authors computed in a previous work ${ }^{30} \Delta \lambda_{P_{\text {control }}}$ for Na-Mmt according to the temperature and adsorption state transitions (Tab. 4). These values are in agreement with the results of Tambach et al. 33 . With the computed bending modulus (and considering that clay layers are 

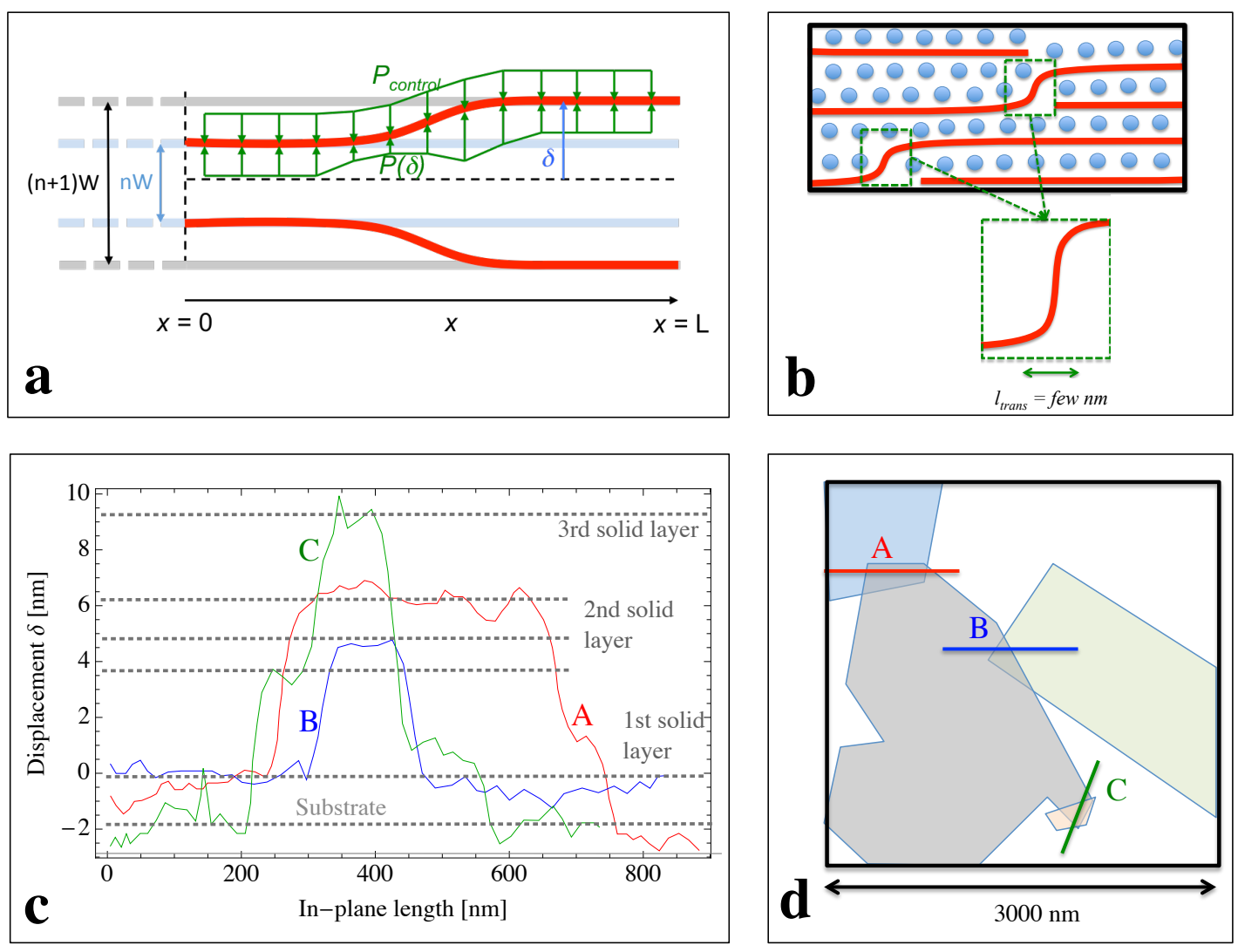

Fig. 9 (a) Sketch of a simple stack of clay layers undergoing a transition of hydration state from $(n+1) W$ to $n W$ state. (b) Sketch of the transition between two hydration states and the superposition of solid layers. (c) Profiles of superposed single-layers of beidelite as reported by Sato et al.1924 and shown in the sketch in (d). An amphiphilic ruthenium(II) complex with length $3.6 \mathrm{~nm}$ is adsorbed on the clay layers and oriented vertically with respect to the basal plane 24 . The reference basal spacing for a configuration with one-layer of such adsorbed complex is about 4-6 nm. 
Table 4 Energy barriers for the hydration transitions in Na-Mmt for a fluid bulk pressure of $10 \mathrm{MPa}$ at the confining pressure corresponding to the stable hydration transitions 30 .

\begin{tabular}{ccc}
\hline Temperature & $0 \mathrm{~W} / 1 \mathrm{~W}$ & $1 \mathrm{~W} / 2 \mathrm{~W}$ \\
\hline $300 \mathrm{~K}$ & $7.25 \mathrm{kT} / \mathrm{nm}^{2}$ & $1.96 \mathrm{kT} / \mathrm{nm}^{2}$ \\
$400 \mathrm{~K}$ & $5.52 \mathrm{kT} / \mathrm{nm}^{2}$ & $1.47 \mathrm{kT} / \mathrm{nm}^{2}$ \\
$500 \mathrm{~K}$ & $4.02 \mathrm{kT} / \mathrm{nm}^{2}$ & $0.61 \mathrm{kT} / \mathrm{nm}^{2}$ \\
\hline rate & $-0.0152 \mathrm{kT} / \mathrm{nm}^{2} / \mathrm{K}$ & $-0.0068 \mathrm{kT} / \mathrm{nm}^{2} / \mathrm{K}$ \\
\hline
\end{tabular}

approximately equidimentional in plan 49 ), $l_{\text {trans }} / L \ll 1$ for NaMmt as soon as $L$ exceeds a few tens of nanometers. So, during the adsorption transitions (Figure9(b)), montmorillonite layers are quite flexible and deform within a bending length $l_{\text {trans }} \sim 1 \mathrm{~nm}$ small compared to their in-plane dimensions L. Hence, the length over which the layers bend during a hydration transition is expected to be in the order of a few nanometers. This result is in agreement with experimental observations (Figure 9 (c) and (d)) of superposed thin beidelite layers $19 \mid 24$ which bend smoothly from the single layer to double or triple layers in transition regions that are less that $50 \mathrm{~nm}$ long.

\subsection{Discussion on the flexibility and microtexture of clays}

The results of the last sections can be used to analyze the flexibility of other layered silicates. Table 5 gathers the in-plane elastic coefficients of the stiffness tensor of some clay minerals. We also compute the bending modulus of these minerals with Eq. 2 accounting for a effective thickness $h_{s}$ corresponding to the reported basal spacing (note that the $h_{s}$ of $9.10 \AA$ is close to the basal spacing in OW state). The values of Young moduli are quite close to the ones associated with Na-Mmt (see Figure 4). The estimated bending moduli have the same order of magnitude. Thus, single layers are expected to present a similar flexural behavior. Therefore, as for Na-Mmt, in a fully exfoliated regime, bent configurations of the single layers of different clay minerals could be achieved by solution stirring.

The behavior of a stack of layers is expected to be much more complex. Actually, the face-to-face cohesion between clay layers depends on the charge density of the individual layers and the nature (notably, valence and size) of counterions. Furthermore, the concentration regime (i.e. dilute or concentrate state) is also a factor to take into account. Figure 10 shows two extreme types of microtextures of clays 10 15: stacked or "brick-like" in which the layers are locally organized in assemblies of thick and rigid crystallites (large coherence length) with large porosity and open texture; and entangled or "sheet-like" with longer flexible layers assembled (low coherence length) with small total porosity and small open porosity (with lenticular pores). These configurations correspond to a concentrated regime. In a dilute regime, layers of lithium $\left(\mathrm{Li}^{+}\right)$-saturated smectites are reported to be isolated ${ }^{10}$; SAXS experiments suggest that exfoliated layers can also be observed in suspensions of $\mathrm{Na}^{+}$exchanged smectites ${ }^{43}$. Stacking of layers shows up from $\mathrm{Na}^{+}$-saturated smectites and increases following the sequence in dilute regime: $\mathrm{Na}^{+}<\mathrm{K}^{+}<\mathrm{Mg}^{2+}<$ $\mathrm{Ca}^{2+}$; with calcium saturated smectite reaching 7 layers 10 . In suspensions, most of the smectites present themselves in the form of
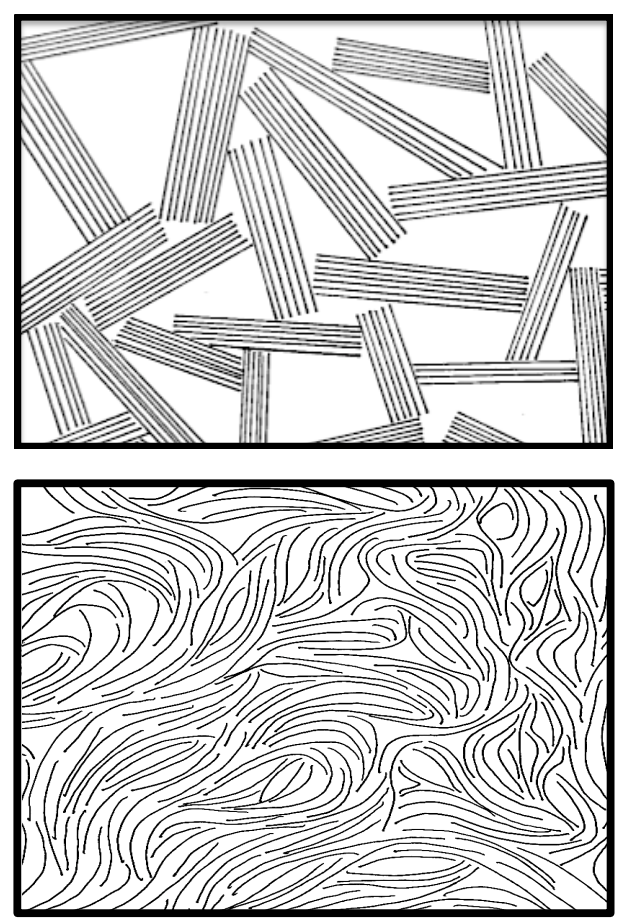

Fig. 10 Representation of the "extreme" microtexture of clays: (top) stacked or "brick-like" and (bottom) entangled or "sheet-like" types 15 .

particles instead of single layers, even in very dilute regimes 10 . Once in the concentrated regime (Fig. 10), the high stiffness of stacks of clay layers makes it unlikely to observe transformations of microstructures from the 'brick-like' to the 'entangled' configuration even under geomechanical loadings. Elastic deformations of stacks with as little as 10 layers are clearly too small to accomodate the entangled state. Plastic deformation could lead to 'entangled' configurations but only if the hydration state is $2 \mathrm{~W}$ or higher. At low hydration, the shear within the stacks is not expected to exceed the yield limit. Accordingly, unless for highly hydrated clays, the microstructure is expected to be a consequence of the early age deposition and depend mostly on the degree of stacking on the dilute regime. For highly hydrated clays, plasticity can lead to significant curvature of the microstructure but is not expected to change the degree of stacking. Therefore, mechanical consolidation is of limited impact on the microstucture and the early age appears critical in the emergence of 'brick-like' or 'entangled' microstructure. Note that the nature of counterions also affects the yield stress of clay particles. The yield stress of Ca-Mmt, for instance, is reported to be higher than Na-Mmt's in $1 \mathrm{~W}$ state and lower than Na-Mmt's in $2 \mathrm{~W}$ and $3 \mathrm{~W}$ states ${ }^{51}$. In $2 \mathrm{~W}$ and 3W cases, the yield stress of Ca-Mmt (2.2 and 2.9 MPa, respectively) is more than twice that of Na-Mmt. Therefore, besides the hydration state, the prevalence of plasticity can well depend on the nature of the swelling clay.

The type of microtexture has important implications in the behavior of clays. The topology of the pores is affected by the flexibility of the layers 69 . The consequences in the effective transfer and mechanical properties of clays are clear. For example, 
Table 5 In-plane components of stiffness tensor $C_{\mathbf{a a a a}}$ and $C_{\mathbf{b b b b}}$ of layered silicates. $D_{\mathbf{a a a a}}$ and $D_{\mathbf{b b b b}}$ are computed with Eq. 2 with an effective thickness $h_{s}$.

\begin{tabular}{|c|c|c|c|c|c|}
\hline Direction & $\begin{array}{l}C_{\text {aaaa }} \\
{[\mathrm{GPa}]}\end{array}$ & $\begin{array}{l}C_{\text {bbbbb }} \\
{[\mathrm{GPa}]}\end{array}$ & $\begin{array}{c}h_{S} \\
{[\AA]}\end{array}$ & $\begin{array}{c}D_{\text {aaaa }} \\
{\left[10^{-17} \text { N.m }\right]}\end{array}$ & $\begin{array}{c}D_{\mathbf{b b b b}} \\
{\left[10^{-17} \text { N.m }\right]}\end{array}$ \\
\hline Na-Mmt (this work) & $247.12 \pm 20.10$ & $276.01 \pm 19.09$ & 9.10 & $2.02 \pm 0.14$ & $1.87 \pm 0.15$ \\
\hline mica 6566 & $147-222$ & $149-178$ & 9.96 & $1.21-1.82$ & $1.22-1.46$ \\
\hline pyrophyllite ${ }^{65}$ & 178.4 & 166.8 & 9.11 & 1.12 & 1.05 \\
\hline dickite 67 & 181.1-184.2 & $178.6-178.8$ & $7.16-7.27$ & $0.554-0.590$ & $0.547-0.573$ \\
\hline kaolinite $67 / 68$ & 164.1-178.5 & $175.5-200.9$ & $7.182-7.587$ & $0.507-0.650$ & $0.542-0.731$ \\
\hline nacrite 67 & $131.8-147.6$ & $157.9-160.8$ & $7.29-7.44$ & $0.425-0.476$ & $0.509-0.552$ \\
\hline
\end{tabular}

highly flexible material with entangled microtexture is reported to present significant resistance to rupture and texture alteration (maybe resulting from irreversible deformations) near rupture, while stacked microtexture presents weaker mechanical properties, due to larger voids inducing stress concentrations, with brittle failure ${ }^{15}$. Regarding the permeability, entangled structures are deemed to be a few orders of magnitude less permeable than stacked structures due to the larger accessible pores in the latter 15 . Similarly, the diffusivity of water and counterions in clays are also expected to be affected by nanotexture $16 \mid 17$.

The flexibility of clay layers and particles is, therefore, a key aspect in the definition of the microtexture of clays. Studies of clays at the mesoscale often adopt the hypothesis of rigid particles 12 70|71. This hypothesis seems reasonable for clays saturated with weakly hydrated ions with high charge and for small particles. Particles with small in-plane dimensions ( $L$ in both $x$ and $y$ directions) are stiffer and larger shear stresses are required to deform them irreversibly. Also, thicker particles, i.e. with more layers in a stack and with higher adsorption state (so, larger pore size), tends to be stiffer albeit more susceptible to the loss of translational correlation due to shear stresses.

\section{Conclusions}

In this paper we computed the bending modulus of Na-Mmt layers and discussed the implications of the flexibility of clay layers in the structuration of clays. Our analysis investigates the case of a single layer and that of a clay particle (stack of hydrated layers). Our main conclusions are:

- The estimation of the bending modulus of Na-Mmt layer by the combination of plate theory with molecular simulations were in agreement with other values reported in the literature $\mathrm{2}^{20 \mid 21}$, notably large scale simulations with explicit solvent 20 . Also, as reported 19 , our estimations showed that a clay layer is more flexible in the direction of unit cell a than in the direction of unit cell $\mathbf{b}$. Estimations of the in-plane Young modulus of clay layers were provided according to an effective thickness corresponding roughly to the thickness of the solid layer plus twice the van der Waals radius of surface oxygen atoms.

- Two regimes of deformation of clay particles are possible depending on the length and number of clay layers: pure bending regime (thin plate theory) for long particles with few layers, or pure shear (derived from thick plate theory) for short particles with many layers. Shearing can become dominant because of the high contrast of stiffness between the solid layers and the pore fluid. The dimensionless quantity $\gamma=\frac{E_{s}}{G_{w}}\left(\frac{h_{\text {tot }}}{L}\right)^{2}$ defines the separation between the two regimes : $\gamma \ll 1$ for pure bending and $\gamma \gg 1$ for pure shear.

- In the analysis of the flexibility of single clay layers and stack of layers (i.e. a clay particle), we showed that, even under stirring, a single clay layer as well as long and thin clay particles may assume a bent configuration. The bending of clay layers has implications in the structuration of these layers and may affect the number of solid layers in a clay particle with translational correlation. Clay particles, as expected, are much stiffer than a single layer, but can still exhibit significant deformation for moderate number of layers $(N<20)$. This is an important information for mesoscale modeling of clays, which in general resort to infinitely rigid particles to represent clay platelets.

- By treating a clay particle as a laminate, in which confined water acts as a soft phase, we showed that for stacks with less than few tens of solid layers, under force control, the shear stress developed in the interlayer pores may exceed the yield stress associated with the inter-layer water. In this case, the shear stresses decrease linearly with the number of solid layers and increase linearly with the applied pressure. Long and thin particles subjected to pressures on the order of the $\mathrm{MPa}$, as the ones found in geomechanics, develop shear stresses on the confined fluid layer that exceed even the yield stresses associated with $1 \mathrm{~W}$ state. This result highlights the role of stacking in enhancing the shear resistance at the particle scale.

- The adsorption state is a key aspect of the flexibility of swelling clay particles. The variations of few Angstroms in the stable basal spacings of $0 \mathrm{~W}, 1 \mathrm{~W} 2 \mathrm{~W}$ and $3 \mathrm{~W}$ states affect the flexibility of the particle. Moreover, the adsorption state influences the yield stress of clays particles. Thus, to a large extent, the stability of a particle under flexural loads depends on the adsorption state.

- The analysis of hydration states transitions points out to the evaluation of the length over which transitions occurs $l_{\text {trans }} \sim \frac{(\Delta \delta)^{2} D}{\Delta \lambda_{P_{\text {control }}}}$, which indicates whether bending is likely to occur. In the case of swelling clays, $l_{\text {trans }}$ is of the order of 
a few $\mathrm{nm}$, that is much smaller than the length of the clay layers. Therefore, hydration transitions in swelling clays involve bending of the solid layers over a length of few $\mathrm{nm}$, which is consistent with experimental observations 19 .

- The bending modulus of single layers of other types of phyllosilicates are similar to the one of Na-Mmt considered here. The face-to-face cohesion of these layers might, however, be very different according to the type of clays and the nature of ions in solution. The variety of the microtexture of clays layers will depend therefore on these aspects. The hypothesis of rigid clay particles might adequately represent the behavior of stacked or brick-like microtextures. The flexibility of the layers plays, though, an important role in entangled microtextures.

A perspective of this work is the development of mesoscale models of clays accounting for the flexibility and hydration transitions in clay particles by means, for instance, of coarse-grained simulations or generalized medium approaches. In particular, the analysis of the behavior of clay samples with evolving lateral dimensions can be assessed taking into account this couplings with possible confrontation with experimental research. Our results can also contribute in the understanding of how the flexibility of clay layers and particles affects phase transitions, associated with gelation or liquid-crystalline formation for example, in the mesoscale $\frac{72}{}$.

\section{Conflicts of interest}

There are no conflicts to declare.

\section{Acknowledgements}

We gratefully acknowledge funding through the project TEAM2ClayDesicc from the French national Research Agency (Agence Nationale de la Recherche contract ANR-14-CE-05-002301).

\section{Notes and references}

1 V. Shenoy and A. Sharma, Journal of the Mechanics and Physics of Solids, 2002, 50, 1155-1173.

2 K. Garikipati, Journal of the Mechanics and Physics of Solids, 2003, 51, 1189-1214.

3 S. Peng and Y. Wei, Journal of the Mechanics and Physics of Solids, 2016, 92, 278-296.

4 I. Benedetti, H. Nguyen, R. A. Soler-Crespo, W. Gao, L. Mao, A. Ghasemi, J. Wen, S. Nguyen and H. D. Espinosa, Journal of the Mechanics and Physics of Solids, 2018, 112, 66-88.

5 J. Paulose, A. S. Meeussen and V. Vitelli, Proceedings of the National Academy of Sciences, 2015, 112, 7639-7644.

6 M. Caruel and L. Truskinovsky, Journal of the Mechanics and Physics of Solids, 2017, 109, 117-141.

7 F. Hubert, L. Caner, A. Meunier and E. Ferrage, American Mineralogist, 2015, 97, 384-398.

8 E. Gartner and H. Hirao, Cement and Concrete Research, 2015, 78, 126-142.

9 T. Honorio and L. Brochard, EAC2 - Second international
RILEM/COST conference on early age cracking and serviceability in cement-based materials and structures (2017), 2017.

10 M. Zabat, R. Harba and H. Van Damme, Colloids and Surfaces A: Physicochemical and Engineering Aspects, 2015, 486, 38-44.

11 L. J. Michot, I. Bihannic, K. Porsch, S. Maddi, C. Baravian, J. Mougel and P. Levitz, Langmuir, 2004, 20, 10829-10837.

12 E. Ferrage, F. Hubert, E. Tertre, A. Delville, L. J. Michot and P. Levitz, Physical Review E, 2015, 91, 062210.

13 M. Delhorme, B. Jönsson and C. Labbez, Soft Matter, 2012, 8, 9691-9704.

14 F. Hetzel, D. Tessier, A.-M. Jaunet and H. Doner, Clays and Clay Minerals, 1994, 42, 242-248.

15 M. Zabat, M. Vayer-Besançon, R. Harba, S. Bonnamy and D. H. V. Damme, Trends in Colloid and Interface Science XI, Steinkopff, 1997, pp. 96-102.

16 S. V. Churakov and T. Gimmi, The Journal of Physical Chemistry C, 2011, 115, 6703-6714.

17 S. V. Churakov, T. Gimmi, T. Unruh, L. R. Van Loon and F. Juranyi, Applied Clay Science, 2014, 96, 36-44.

18 F. Salles, O. Bildstein, J. M. Douillard, M. Jullien, J. Raynal and H. Van Damme, Langmuir, 2010, 26, 5028-5037.

19 H. Sato, A. Yamagishi and K. Kawamura, The Journal of Physical Chemistry B, 2001, 105, 7990-7997.

20 J. L. Suter, P. V. Coveney, H. C. Greenwell and M.-A. Thyveetil, The Journal of Physical Chemistry C, 2007, 111, 8248-8259.

21 O. L. Manevitch and G. C. Rutledge, The Journal of Physical Chemistry B, 2004, 108, 1428-1435.

22 J. L. Suter and P. V. Coveney, Soft Matter, 2009, 5, 3896-3904.

23 J. L. Suter and P. V. Coveney, Soft Matter, 2009, 5, 2239-2251.

24 K. Tamura, H. Setsuda, M. Taniguchi and A. Yamagishi, Langmuir, 1999, 15, 6915-6920.

25 I. Benichou and S. Givli, Journal of the Mechanics and Physics of Solids, 2013, 61, 94-113.

26 I. Benichou and S. Givli, Physical Review Letters, 2015, 114, 095504.

27 I. Benichou, Z. Yaojun, O. Dudko and S. Givli, Journal of the Mechanics and Physics of Solids, 2016, 95, 44-63.

28 Z. P. Bažant and M. Z. Bazant, Journal of the Mechanics and Physics of Solids, 2012, 60, 1644-1659.

29 M. Z. Bazant and Z. P. Bažant, Journal of the Mechanics and Physics of Solids, 2012, 60, 1660-1675.

30 T. Honorio, L. Brochard and M. Vandamme, Langmuir, 2017, 33, 12766-12776.

31 E. Ferrage, B. Lanson, N. Malikova, A. Plançon, B. A. Sakharov and V. A. Drits, Chemistry of Materials, 2005, 17, 3499-3512.

32 E. Ferrage, Clays and Clay Minerals, 2016, 64, 348-373.

33 T. J. Tambach, P. G. Bolhuis, E. J. M. Hensen and B. Smit, Langmuir, 2006, 22, 1223-1234.

34 R. M. Shroll and D. E. Smith, The Journal of Chemical Physics, 1999, 111, 9025-9033.

35 D. E. Smith, Y. Wang, A. Chaturvedi and H. D. Whitley, The Journal of Physical Chemistry B, 2006, 110, 20046-20054.

36 Y.-c. Chen, E. Fried and D. A. Tortorelli, Journal of the Mechanics and Physics of Solids, 2012, 60, 904-920. 
37 V. Drits, D. Eberl and J. Srodon, Clays and Clay Minerals, 1998, 46, 38-50.

38 K. Mystkowski, J. Srodon and F. Elsass, Clay Minerals, 2000, 35, 545-557.

39 E. C. Jonas and R. M. Oliver, Clays Clay Miner, 1967, 15, 2733.

40 T. Beermann and O. Brockamp, Clay Minerals, 2016, 40, 113.

41 P. H. Nadeau, CLAY MINER. Clay Miner., 1985, 20, 499.

42 D. G. H. Ballard and G. R. Rideal, Journal of Materials Science, 1983, 18, 545-561.

43 M. Morvan, D. Espinat, J. Lambard and T. Zemb, Colloids and Surfaces A: Physicochemical and Engineering Aspects, 1994, 82, 193-203.

44 I. Bihannic, D. Tchoubar, S. Lyonnard, G. Besson and F. Thomas, Journal of Colloid and Interface Science, 2001, 240, 211-218.

45 J. L. Lutkenhaus, E. A. Olivetti, E. A. Verploegen, B. M. Cord, D. R. Sadoway and P. T. Hammond, Langmuir, 2007, 23, 8515-8521.

46 E. L. Hansen, H. Hemmen, D. M. Fonseca, C. Coutant, K. D. Knudsen, T. S. Plivelic, D. Bonn and J. O. Fossum, Nature, Scientific Reports, 2012, 2, year.

47 M. X. Reinholdt, F. Hubert, M. Faurel, E. Tertre, A. Razafitianamaharavo, G. Francius, D. Prêt, S. Petit, E. Béré, M. Pelletier and E. Ferrage, Applied Clay Science, 2013, 77, 18-32.

48 Z. Zhang and J. S. v. Duijneveldt, Soft Matter, 2007, 3, 596604.

49 D. J. Greenland and M. H. B. Hayes, The chemistry of soil constituents., 2016.

50 J. H. Lee and S. Guggenheim, American Mineralogist, 1981, 66, 350-357.

51 B. Carrier, $P h D$ Thesis, Université Paris-Est, 2013.

52 W. Loewenstein, American Mineralogist, 1954, 39, 92-96.

53 B. F. Ngouana W. and A. G. Kalinichev, The Journal of Physical Chemistry C, 2014, 118, 12758-12773.

54 S. Plimpton, Journal of Computational Physics, 1995, 117, 119.

55 R. T. Cygan, J.-J. Liang and A. G. Kalinichev, The Journal of
Physical Chemistry B, 2004, 108, 1255-1266.

56 B. Carrier, M. Vandamme, R. J.-M. Pellenq and H. Van Damme, The Journal of Physical Chemistry C, 2014, 118, 8933-8943.

57 M. A. Mazo, L. I. Manevitch, E. B. Gusarova, A. A. Berlin, N. K. Balabaev and G. C. Rutledge, The Journal of Physical Chemistry C, 2008, 112, 17056-17062.

58 Z. Bažant and L. Cedolin, Stability of Structures: Elastic, Inelastic, Fracture, and Damage Theories, Dover Publications, 1991.

59 D. H. Everett, Basic principles of colloid science, 1988, http: //cds.cern.ch/record/1619422

60 U. Raviv, S. Perkin, P. Laurat and J. Klein, Langmuir, 2004, 20, 5322-5332.

61 L. Brochard, T. Honorio, M. Vandamme, M. Bornert and M. Peigney, Acta Geotechnica, 2017, 1-19.

62 D. A. Laird and C. Shang, Clays and Clay Minerals, 1997, 45, 681-689.

63 K. Verburg, P. Baveye and M. B. McBride, Soil Science Society of America Journal, 1995, 59, 1268-1273.

64 E. S. Boek, P. V. Coveney and N. T. Skipper, Langmuir, 1995, 11, 4629-4631.

65 G. D. Zartman, H. Liu, B. Akdim, R. Pachter and H. Heinz, The Journal of Physical Chemistry C, 2010, 114, 1763-1772.

66 S. L. Teich-McGoldrick, J. A. Greathouse and R. T. Cygan, The Journal of Physical Chemistry C, 2012, 116, 15099-15107.

67 B. Militzer, H.-R. Wenk, S. Stackhouse and L. Stixrude, American Mineralogist, 2015, 96, 125-137.

68 H. Sato, K. Ono, C. T. Johnston and A. Yamagishi, American Mineralogist, 2015, 90, 1824-1826.

69 H. Van Damme, P. Levitz, J. J. Fripiat, J. F. Alcover, L. Gatineau and F. Bergaya, Physics of Finely Divided Matter, Springer Berlin Heidelberg, 1985, pp. 24-30.

70 D. Ebrahimi, A. J. Whittle and R. J.-M. Pellenq, The Journal of Chemical Physics, 2014, 140, 154309.

71 D. Ebrahimi, R. J.-M. Pellenq and A. J. Whittle, Granular Matter, 2016, 18, 49.

72 L. J. Michot, I. Bihannic, S. Maddi, S. S. Funari, C. Baravian, P. Levitz and P. Davidson, Proceedings of the National Academy of Sciences, 2006, 103, 16101-16104. 